\title{
Sparse quadratic regulator
}

\author{
Mihailo R. Jovanović and Fu Lin
}

\begin{abstract}
We consider a control design problem aimed at balancing quadratic performance of linear systems with additional requirements on the control signal. These are introduced in order to obtain controls that are either sparse or infrequently changing in time. To achieve this objective, we augment a standard quadratic performance index with an additional term that penalizes either the $\ell_{1}$ norm or the total variation of the control signal. We show that the minimizer of this convex optimization problem can be found by solving a two point boundary value problem (TPBVP) with non-differentiable nonlinearities. Furthermore, we employ alternating direction method of multipliers to determine the optimal controller iteratively from a sequence of linear TPBVPs. Examples are provided to illustrate the developed method.

Index Terms-Alternating direction method of multipliers, convex optimization, linear time-invariant systems, quadratic performance, sparsity, total variation.
\end{abstract}

\section{INTRODUCTION}

Linear quadratic regulator (LQR) theory has a rich history and it represents a corner stone of modern systems theory. In addition to finding use in a number of applications, its popularity stems from elegant theoretical framework that provides performance guarantees and computationally efficient means for forming control action from available measurements.

In applications, it is often desired to have control inputs that are either sparse or infrequently changing in time. In multi-period investments [1], for example, there is a transaction cost associated with trades, i.e., buying or selling assets. Thus, limiting the number of trades to reduce the transaction cost imposes sparsity constraint on the control input. In temperature control of energy-efficient buildings [2], it is of interest to shut down the air-conditioners over a period of time to reduce energy consumption and to increase the longevity of the compressors. In this case, a sparse control signal implies that the compressors are turned off for most of the time.

This paper combines standard control theory with tools from cardinality minimization and compressive sensing to design sparse and infrequently changing control inputs that minimize quadratic performance of linear-time invariant systems. Desired properties are enforced by augmenting a standard quadratic performance index with additional terms that penalize either the $\ell_{1}$

Financial support from the National Science Foundation under Award CMMI-09-27720 and from the University of Minnesota Initiative for Renewable Energy and the Environment under Early Career Award RC-0014-11 is gratefully acknowledged.

M. R. Jovanović and F. Lin are with the Department of Electrical and Computer Engineering, University of Minnesota, Minneapolis, MN 55455. E-mails: mihailo@umn.edu, fu@umn.edu. norm or the total variation of the control signal. We show that the minimizer of this convex optimization problem can be found by solving a TPBVP with nondifferentiable nonlinearities. Furthermore, we utilize the alternating direction method of multipliers to determine the optimal controller iteratively from a sequence of linear TPBVPs. Examples are provided to illustrate the developed method.

Developing efficient algorithms for a closely related problem, namely model predictive control (MPC), has been an active research topic [3]-[11]. It was shown in [3], [4], [8] that customized interior point methods that exploit sparsity structures are very efficient for MPC. Recently, the alternating direction method of multipliers (ADMM) [12] has been employed by several authors to develop distributed implementation for MPC [9]-[11]. For continuous-time systems, we employ the ADMM algorithm and the soft thresholding operator to handle the non-differentiable nonlinearities. This approach allows us to convert nonlinear TPBVPs into a sequence of linear TPBVPs.

In controls community, ADMM has recently been used to design sparse feedback gains that strike a balance between the performance of distributed systems and the sparsity of the controller [13], [14], to select leaders in stochastically forced dynamic networks [15], [16], to identify sparse representation of consensus networks [17], to counteract spread of misinformation in social networks [18], and to design sparse wide-area controllers in power networks [19]. In addition, ADMM has been found useful in distributed implementation of MPC [11] and total variation regularized MPC [20].

Our presentation is organized as follows. In Section II, we formulate the quadratic regulator problem with $\ell_{1}$ and total variation regularization terms for continuoustime linear time-invariant systems. In Section III, we show that the necessary conditions for optimality take the form of nonlinear TPBVPs. In Section IV, we employ the ADMM algorithm to convert the nonlinear TPBVPs into a sequence of linear TPBVPs. We provide two illustrative examples in Section V and conclude the paper with a summary of our contributions in Section VI.

\section{Problem formulation}

We consider linear time-invariant systems

$$
\dot{x}=A x+B u, \quad t \in[0, T], \quad x(0)=x_{0}
$$

with $n$ states and $m$ control inputs. The objective is to design a control input $u$ on the time interval $[0, T]$ to 
minimize

$$
J(x, u, \dot{u})=J_{\mathrm{lqr}}(x, u)+\gamma \int_{0}^{T} g(u(t), \dot{u}(t)) \mathrm{d} t .
$$

Here, $\gamma$ is a positive parameter and $g$ is a function that penalizes the $\ell_{1}$ norm of the vector $u(t)$,

$$
g(u(t))=\|u(t)\|_{\ell_{1}}=\sum_{i=1}^{m}\left|u_{i}(t)\right|
$$

or the $\ell_{1}$ norm of the vector $\dot{u}(t)$,

$$
g(\dot{u}(t))=\|\dot{u}(t)\|_{\ell_{1}}=\sum_{i=1}^{m}\left|\dot{u}_{i}(t)\right| .
$$

The $\ell_{1}$ norms of $u(t)$ and $\dot{u}(t)$ are introduced in order to induce a sparse control input and a control input with small total variation, respectively. Recently, the $\ell_{1}$ norm has been used as a proxy for promoting sparsity in optimization variables [21, Chapter 6]; also see [22] for alternative sparsity-promoting penalty functions. by

The quadratic performance index $J_{\text {lqr }}$ in (1) is given $J_{\mathrm{lqr}}(x, u)=\frac{1}{2}\left(\langle x, Q x\rangle+\langle u, R u\rangle+x^{T}(T) Q_{f} x(T)\right)$ with

$$
\langle x, Q x\rangle:=\int_{0}^{T} x^{T}(t) Q x(t) \mathrm{d} t
$$

and the standard assumptions on the state, terminal, and control cost matrices

$$
Q=Q^{T} \geq 0, Q_{f}=Q_{f}^{T} \geq 0, R=R^{T}>0 .
$$

By introducing an additional optimization variable $v$, optimal control problem (1) can be equivalently formulated as

$$
\begin{array}{cl}
\underset{x, u, v}{\operatorname{minimize}} & J_{\mathrm{lqr}}(x, u)+\gamma \int_{0}^{T}\|v(t)\|_{\ell_{1}} \mathrm{~d} t \\
\text { subject to } & A x(t)+B u(t)-\dot{x}(t)=0 \\
& {[L u](t)-v(t)=0} \\
& x(0)=x_{0}, \quad t \in[0, T]
\end{array}
$$

where the action of a linear operator $L$ on the control signal $u$ is determined by

$$
[L u](t):= \begin{cases}u(t) & \text { for }(\mathrm{SP}) \\ \dot{u}(t) & \text { for }(\mathrm{TV})\end{cases}
$$

Problem (2) is a convex optimization problem with a convex objective function and linear constraints. Clearly, for $\gamma=0$, it simplifies to the standard LQR problem [23].

\section{CONDITIONS FOR OPTIMALITY}

We next show that the necessary and sufficient conditions for optimality of (2) are given by

$$
\begin{aligned}
\dot{x}(t) & =A x(t)+B u(t), \quad x(0)=x_{0} \\
\dot{y}(t) & =-Q x(t)-A^{T} y(t), \quad y(T)=Q_{f} x(T) \\
0 & =R u(t)+B^{T} y(t)+\left[L^{*} z\right](t) \\
0 & =[L u](t)-v(t) \\
0 & \in \gamma \partial\|v(t)\|_{\ell_{1}}-z(t) .
\end{aligned}
$$

Here, $L^{*}$ is the adjoint of the operator $L$, and $\partial$ is the subdifferential operator, with the $i$ th component of the vector

$$
\partial\|v(t)\|_{\ell_{1}}:=\left[\begin{array}{lll}
\partial\left|v_{1}(t)\right| & \cdots & \partial\left|v_{m}(t)\right|
\end{array}\right]^{T}
$$

determined by

$$
\partial\left|v_{i}(t)\right|= \begin{cases}1, & v_{i}(t)>0 \\ {[-1,1],} & v_{i}(t)=0 \\ -1, & v_{i}(t)<0\end{cases}
$$

To derive (4), we form the Lagrangian associated with the constrained optimal control problem (2),

$$
\begin{aligned}
& \mathcal{L}(x, y ; u, v, z)=J_{\mathrm{lqr}}(x, u)+\gamma \int_{0}^{T}\|v(t)\|_{\ell_{1}} \mathrm{~d} t+ \\
& \langle y, A x+B u-\dot{x}\rangle+\langle z, L u-v\rangle
\end{aligned}
$$

where $y$ and $z$ are the Lagrange multipliers associated with the linear constraints in (2). Taking variation of $\mathcal{L}$ with respect to the Lagrange multiplier $y$ and the state trajectory $x$ yields the standard TPBVP for the states of the original and the adjoint systems [23]

$$
\begin{array}{ll}
\dot{x}(t)=A x(t)+B u(t), & x(0)=x_{0} \\
\dot{y}(t)=-Q x(t)-A^{T} y(t), & y(T)=Q_{f} x(T) .
\end{array}
$$

On the other hand, setting variations of $\mathcal{L}$ with respect to $u, z$, and $v$ to zero, respectively, leads to

$$
\begin{aligned}
& 0=R u(t)+B^{T} y(t)+\left[L^{*} z\right](t) \\
& 0=[L u](t)-v(t) \\
& 0 \in \gamma \partial\|v(t)\|_{\ell_{1}}-z(t) .
\end{aligned}
$$

\section{A. $\ell_{1}$-regularized optimal control problem}

For the sparsity-promoting optimal control problem (1)-(SP), the linear operator $L$ in (2) is the identity operator, and the necessary conditions for optimality (7) simplify to

$$
0 \in R u(t)+B^{T} y(t)+\gamma \partial\|u(t)\|_{\ell_{1}} .
$$

In addition, when the control cost is the scaled identity, $R:=r I$ with $r>0$, we can obtain an explicit expression for the control input $u(t)$,

$$
\begin{aligned}
u(t) & =-(1 / r) S_{\gamma}\left(B^{T} y(t)\right) \\
& =-S_{\gamma / r}\left((1 / r) B^{T} y(t)\right)
\end{aligned}
$$




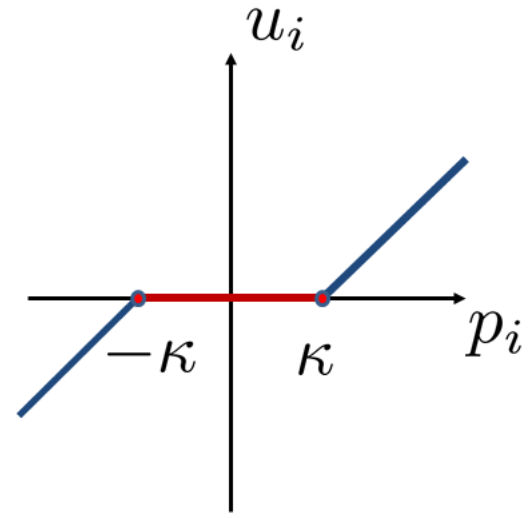

Fig. 1. The action of the soft thresholding operator $S_{\kappa}(\cdot)$ on the $i$ th component of the vector $p$.

where the action of the soft thresholding operator $S_{\kappa}(\cdot)$, with $\kappa=\gamma / r$, on the $i$ th component of the vector $p(t):=(1 / r) B^{T} y(t)$ is given by

$$
S_{\kappa}\left(p_{i}\right)= \begin{cases}p_{i}-\kappa, & p_{i}>\kappa \\ 0, & p_{i} \in[-\kappa, \kappa] \\ p_{i}+\kappa, & p_{i}<-\kappa .\end{cases}
$$

Thus, the optimal control input for the sparsitypromoting optimal control problem (1)-(SP) with $R:=$ $r I$ can be obtained from the solution of the following nonlinear TPBVP

$$
\begin{aligned}
& {\left[\begin{array}{l}
\dot{x}(t) \\
\dot{y}(t)
\end{array}\right]=\left[\begin{array}{cc}
A & 0 \\
-Q & -A^{T}
\end{array}\right]\left[\begin{array}{l}
x(t) \\
y(t)
\end{array}\right]-} \\
& {\left[\begin{array}{c}
B \\
0
\end{array}\right] S_{\gamma / r}\left((1 / r) B^{T} y(t)\right)} \\
& u(t)=-S_{\gamma / r}\left((1 / r) B^{T} y(t)\right) \\
& {\left[\begin{array}{c}
x_{0} \\
0
\end{array}\right]=\left[\begin{array}{ll}
I & 0 \\
0 & 0
\end{array}\right]\left[\begin{array}{l}
x(0) \\
y(0)
\end{array}\right]+} \\
& {\left[\begin{array}{cc}
0 & 0 \\
Q_{f} & -I
\end{array}\right]\left[\begin{array}{l}
x(T) \\
y(T)
\end{array}\right] .}
\end{aligned}
$$

By comparing the expression for the control input in (10) with the expression for the control input in LQR,

$$
u_{\mathrm{lqr}}(t)=-(1 / r) B^{T} y(t)
$$

we see that sparsity is enforced by passing the vector $p(t)=(1 / r) B^{T} y(t)$ through a dead-zone nonlinearity shown in Fig. 1 (with $\kappa=\gamma / r$ ). If $p_{i}(t) \in[-\gamma / r, \gamma / r]$, $u_{i}(t)$ is set to zero; if $\left|p_{i}(t)\right|>\gamma / r, u_{i}(t)$ is obtained by moving $p_{i}(t)$ towards zero with the amount $\gamma / r$. This demonstrates that larger values of $\gamma$ and smaller values of control penalty $r$ encourage sparser control signals.

System (10) can be solved using MATLAB's TPBVP solver bvp4c or the Chebfun computing environment [24]; in fact, we found it most convenient to use bvp4c in conjunction with Chebfun.
Remark 1 (Block sparsity): Rather than enforcing sparsity by driving individual components of the control signal to zero (at different time intervals), it may be of interest to drive all components of the control signal to zero at certain time intervals. For example, this may be relevant in applications where all actuators in a system should either be on or off. Block sparsity can be enforced by replacing the $\ell_{1}$ norm of the vector $u(t)$ in the definition of the function $g$ appearing in (2) with the Euclidean norm of $u(t)$ (e.g., see [12]),

$$
g(u(t))=\|u(t)\|_{2}=\sqrt{\sum_{i=1}^{m} u_{i}^{2}(t)} .
$$

In the TPBVP (10), soft thresholding operator should be replaced by block soft thresholding operator [12] whose action on the vector $p(t)=(1 / r) B^{T} y(t)$ is given by

$S_{\kappa}^{\text {block }}(p(t))= \begin{cases}\left(1-\frac{\kappa}{\|p(t)\|_{2}}\right) p(t), & \|p(t)\|_{2}>\kappa \\ 0, & \|p(t)\|_{2} \leq \kappa\end{cases}$

with $\kappa=\gamma / r$.

\section{B. Total variation regularized optimal control problem}

In applications, it is also of interest to promote sparsity with respect to the rate of change in the control input. This objective can be attained by incorporating the total variation of $u$ into the objective function. In this case, $g$ is given by (TV), and the linear operator $L$ in (2) becomes the differential operator.

We next show that the conditions for optimality (4) for the optimal control problem (2) with total variation regularization term (TV) simplify to

$$
\begin{aligned}
{\left[\begin{array}{c}
\dot{x}(t) \\
\dot{y}(t)
\end{array}\right]=} & {\left[\begin{array}{cc}
A & 0 \\
-Q & -A^{T}
\end{array}\right]\left[\begin{array}{l}
x(t) \\
y(t)
\end{array}\right]-\left[\begin{array}{c}
B \\
0
\end{array}\right] u(t) } \\
\dot{z}(t)= & R u(t)+B^{T} y(t) \\
\dot{u}(t)= & \operatorname{Jump}_{\gamma}(z(t)) \\
{\left[\begin{array}{c}
x_{0} \\
0
\end{array}\right]=} & {\left[\begin{array}{ll}
I & 0 \\
0 & 0
\end{array}\right]\left[\begin{array}{l}
x(0) \\
y(0)
\end{array}\right]+} \\
& {\left[\begin{array}{cc}
0 & 0 \\
Q_{f} & -I
\end{array}\right]\left[\begin{array}{l}
x(T) \\
y(T)
\end{array}\right] } \\
z(0)= & z(T)=0 .
\end{aligned}
$$

Here, the action of the jump operator $\operatorname{Jump}_{\gamma}(\cdot)$ on the $i$ th component of the vector $z$ is given by

$$
\operatorname{Jump}_{\gamma}\left(z_{i}\right):=\left\{\begin{aligned}
\infty, & z_{i}>\gamma \\
0, & z_{i} \in[-\gamma, \gamma] \\
-\infty, & z_{i}<-\gamma .
\end{aligned}\right.
$$

To derive (11), we take variation of $\mathcal{L}$ with respect to $u$,

$$
0=\left\langle R u+B^{T} y, \tilde{u}\right\rangle+\langle z, \dot{\tilde{u}}\rangle
$$


and use integration by parts to obtain

$0=\left\langle R u+B^{T} y-\dot{z}, \tilde{u}\right\rangle+z^{T}(T) \tilde{u}(T)-z^{T}(0) \tilde{u}(0)$.

Since this condition has to hold for all variations $\tilde{u}$, we have

$$
\dot{z}(t)=R u(t)+B^{T} y(t)
$$

with the boundary conditions

$$
z(0)=z(T)=0 .
$$

On the other hand, taking subdifferential of $\mathcal{L}$ with respect to $v$ yields

$$
\gamma \partial\|v(t)\|_{\ell_{1}}-z(t)=0
$$

which consequently leads to

$$
v(t)=\operatorname{Jump}_{\gamma}(z(t)) .
$$

The derivation of (13) will be reported elsewhere.

The jump operator (12) introduces discontinuity, and hence, difficulty in solving the TPBVP (11). This challenge can be overcome by augmenting the objective function (1) with an additional term, $(\delta / 2)\langle\dot{u}, \dot{u}\rangle$, where $\delta$ is a small positive parameter. The quadratic regularization on $v:=\dot{u}$ introduces the following continuos approximation of the jump operator (12)

$$
\operatorname{Jump}_{\gamma}(z) \approx \frac{1}{\delta} S_{\gamma}(z), \quad 0<\delta \ll 1
$$

where $S_{\gamma}(\cdot)$ is the soft-thresholding operator (9).

\section{Alternating Direction method of MULTIPLIERS}

We next use the alternating direction method of multipliers (ADMM) to solve the finite horizon optimal control problem (2) with $L$ in (3). We refer the reader to a recent paper [12] that provides a survey of the utility of ADMM in distributed optimization and modern applications.

The procedure presented in Section II leads to nonlinear TPBVPs (10) and (11) for the linear quadratic regulator problem augmented by the $\ell_{1}$ and the total variation regularization terms, respectively. In contrast, ADMM provides an alternative approach that computes the optimal controller by solving a sequence of linear TPBVPs. As a result, this algorithm is well-suited for efficient implementation for problems with a large number of optimization variables.

We begin by introducing the augmented Lagrangian

$$
\begin{aligned}
\mathcal{L}_{\rho}(x, y ; u, v, z):= & \mathcal{L}(x, y ; u, v, z)+ \\
& \frac{\rho}{2}\langle L u-v, L u-v\rangle
\end{aligned}
$$

where $\rho$ is a positive parameter and $\mathcal{L}$ is the Lagrangian given by (5). For the total variation problem, we will show that the quadratic penalty on the difference between $\dot{u}$ and $v$ allows us to avoid issues that we encountered with the jump operator in Section II.

Taking variation of $\mathcal{L}_{\rho}$ with respect to $y$ and $x$ yields the TPBVP (6). On the other hand, we will determine $u, v$, and $z$ from the following sequence of iterations

$$
\begin{aligned}
u^{k+1}(t) & :=\underset{u}{\arg \min } \mathcal{L}_{\rho}\left(\cdot ; u, v^{k}, z^{k}\right) \\
v^{k+1}(t) & :=\underset{v}{\arg \min } \mathcal{L}_{\rho}\left(\cdot ; u^{k+1}, v, z^{k}\right) \\
z^{k+1}(t) & :=z^{k}(t)+\rho\left(\left[L u^{k+1}\right](t)-v^{k+1}(t)\right)
\end{aligned}
$$

until

$$
\begin{aligned}
\left\langle L u^{k+1}-v^{k+1}, L u^{k+1}-v^{k+1}\right\rangle & \leq \epsilon^{2} \\
\left\langle v^{k+1}-v^{k}, v^{k+1}-v^{k}\right\rangle & \leq \epsilon^{2} .
\end{aligned}
$$

The necessary conditions for the optimality of the $u$-minimization problem (15a) and the $v$-minimization problem (15b) are given by (17). Here, the $u$ minimization problem (15a) can be solved efficiently using the bvp4c in conjunction with Chebfun. On the other hand, the solution to the $v$-minimization problem (15b), for both sparsity-promoting and total variation problems, amounts to a soft-thresholding operation (9).

To derive (17), we take variation $\tilde{u}$ of $\mathcal{L}_{\rho}$ with respect to $u$ and keep the linear term in $\tilde{u}$ to get

$$
\begin{aligned}
0 & =\mathcal{L}_{\rho}(x, y ; u+\tilde{u}, v, z)-\mathcal{L}_{\rho}(x, y ; u, v, z) \\
& =\left\langle R u+B^{T} y+\rho L^{*}\left(L u-w^{k}\right), \tilde{u}\right\rangle
\end{aligned}
$$

where

$$
w^{k}(t):=v^{k}(t)-(1 / \rho) z^{k}(t) .
$$

Since the above condition has to hold for all variations $\tilde{u}$ we have

$$
\left(R+\rho L^{*} L\right) u=-B^{T} y+\rho L^{*} w^{k} .
$$

Moreover, taking subdifferential of $\mathcal{L}_{\rho}$ with respect to $v$ yields

$\gamma \partial\|v(t)\|_{\ell_{1}}+\rho v(t)=\rho\left(\left[L u^{k+1}\right](t)+(1 / \rho) z^{k}(t)\right)$

which consequently leads to

$$
v^{k+1}(t)=S_{\gamma / \rho}\left(\left[L u^{k+1}\right](t)+(1 / \rho) z^{k}(t)\right) .
$$

For the $\ell_{1}$-regularized optimal control problem (2)(SP), $L$ is the identity operator, and the equation for $u^{k+1}$ in (17) simplifies to

$$
u^{k+1}(t)=(R+\rho I)^{-1}\left(-B^{T} y^{k+1}(t)+\rho w^{k}(t)\right) .
$$

For the optimal control problem (2)-(TV), $L$ is the differential operator, and the equation for $u^{k+1}$ in (17) simplifies to

$\ddot{u}^{k+1}(t)=\dot{w}^{k}(t)+(1 / \rho)\left(R u^{k+1}(t)+B^{T} y^{k+1}(t)\right)$

with the boundary conditions

$$
\dot{u}^{k+1}(0)=w^{k}(0), \quad \dot{u}^{k+1}(T)=w^{k}(T) .
$$

Therefore, for the quadratic regulator problem with total variation regularization term, TPBVP (17) resulting from the use of ADMM can be rewritten as (18). 


$$
\begin{gathered}
u:\left\{\begin{array}{c}
\dot{x}^{k+1}(t) \\
\dot{y}^{k+1}(t)
\end{array}\right]=\left[\begin{array}{cc}
A & 0 \\
-Q & -A^{T}
\end{array}\right]\left[\begin{array}{l}
x^{k+1}(t) \\
y^{k+1}(t)
\end{array}\right]+\left[\begin{array}{c}
B \\
0
\end{array}\right] u^{k+1}(t) \\
{\left[\left(R+\rho L^{*} L\right) u^{k+1}\right](t)=-B^{T} y^{k+1}(t)+\rho\left[L^{*}\left(v^{k}-(1 / \rho) z^{k}\right)\right](t)} \\
{\left[\begin{array}{c}
x_{0} \\
0
\end{array}\right]=\left[\begin{array}{ll}
I & 0 \\
0 & 0
\end{array}\right]\left[\begin{array}{l}
x^{k+1}(0) \\
y^{k+1}(0)
\end{array}\right]+\left[\begin{array}{cc}
0 & 0 \\
Q_{f} & -I
\end{array}\right]\left[\begin{array}{l}
x^{k+1}(T) \\
y^{k+1}(T)
\end{array}\right]} \\
v: \quad v^{k+1}(t)=S_{\gamma / \rho}\left(\left[L u^{k+1}\right](t)+(1 / \rho) z^{k}(t)\right) \\
z: \quad z^{k+1}(t)=z^{k}(t)+\rho\left(\left[L u^{k+1}\right](t)-v^{k+1}(t)\right)
\end{gathered}
$$

$$
\begin{aligned}
u:\left\{\begin{aligned}
{\left[\begin{array}{c}
\dot{x}^{k+1}(t) \\
\dot{y}^{k+1}(t)
\end{array}\right] } & =\left[\begin{array}{cc}
A & 0 \\
-Q & -A^{T}
\end{array}\right]\left[\begin{array}{l}
x^{k+1}(t) \\
y^{k+1}(t)
\end{array}\right]+\left[\begin{array}{c}
B \\
0
\end{array}\right] u^{k+1}(t) \\
\dot{u}^{k+1}(t) & =\xi^{k+1}(t) \\
\dot{\xi}^{k+1}(t) & =(1 / \rho)\left(R u^{k+1}(t)+B^{T} y^{k+1}(t)\right)+\dot{v}^{k}(t)-(1 / \rho) \dot{z}^{k}(t) \\
{\left[\begin{array}{c}
x_{0} \\
0
\end{array}\right] } & =\left[\begin{array}{ll}
I & 0 \\
0 & 0
\end{array}\right]\left[\begin{array}{l}
x^{k+1}(0) \\
y^{k+1}(0)
\end{array}\right]+\left[\begin{array}{cc}
0 & 0 \\
Q_{f} & -I
\end{array}\right]\left[\begin{array}{c}
x^{k+1}(T) \\
y^{k+1}(T)
\end{array}\right] \\
\dot{u}^{k+1}(0) & =v^{k}(0)-(1 / \rho) z^{k}(0) \\
\dot{u}^{k+1}(T) & =v^{k}(T)-(1 / \rho) z^{k}(T)
\end{aligned}\right. \\
v: \quad v^{k+1}(t)=S_{\gamma / \rho}\left(\dot{u}^{k+1}(t)+(1 / \rho) z^{k}(t)\right) \\
z: \quad z^{k+1}(t)=z^{k}(t)+\rho\left(\dot{u}^{k+1}(t)-v^{k+1}(t)\right)
\end{aligned}
$$

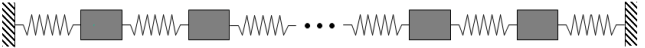

Fig. 2. Mass-spring system.

\section{EXAMPLES}

\section{A. Mass-spring system}

For a mass-spring system on a line shown in Fig. 2 with $N=10$ masses, we set all masses and spring constants to unity and select $Q=I, R=I$, and $Q_{f}=q_{f} I$ with $q_{f}=10^{4}$. We first solve the optimal control problem (2) with the $\ell_{1}$ penalty (SP) on the control input. Optimal control and position trajectories for the mass indexed by $n=6$ are obtained by solving TPBVP (10) using bvp4c in conjunction with Chebfun and they are shown in Fig. 3. Note that as $\gamma$ increases, the sparsity of the control signal $u_{6}(t)$ increases, at the expense of a larger deviation of position $p_{6}(t)$.

For the total variation penalty (TV), the optimal control and position trajectories are obtained by solving TPBVP (11) with the jump operator approximated by (14); see Fig. 4. As $\gamma$ increases, we see that the total variation of the control signal $u_{6}(t)$ decreases, at the expense of a larger deviation of $p_{6}(t)$.

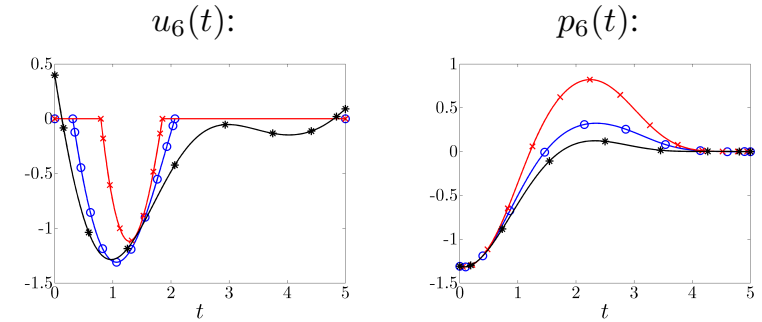

Fig. 3. Optimal control and position trajectories for the mass indexed by $n=6$ in a mass-spring system with $N=10$ masses and $\gamma=1$ $(\circ), \gamma=10(\times)$, and standard LQR $(*)$.

\section{B. Inverted pendulum}

The linearized model of an inverted pendulum on a cart is given by

$$
\begin{aligned}
& \ddot{p}=-\frac{m g}{M} \theta+\frac{1}{M} u \\
& \ddot{\theta}=\frac{(M+m) g}{2 M l} \theta-\frac{1}{2 M l} u .
\end{aligned}
$$

Here, $p$ is the cart position, $\theta$ is the pendulum angle deviation from the upright position, $u$ is the force applied on the cart, $m$ is the pendulum mass, $2 l$ is the pendulum length, and $M$ is the cart mass. We set $M=m=$ $2 l=1, g=9.81$, and select the initial condition $x_{0}=$ $\left[\begin{array}{llll}0 & \pi / 10 & 0 & 0\end{array}\right]^{T}$ and weight matrices $\{Q=R=I$, $\left.Q_{f}=q_{f} I\right\}$ with $q_{f}=1$.

We solve the optimal control problem (2) with the total variation penalty (TV) on the control input. The 

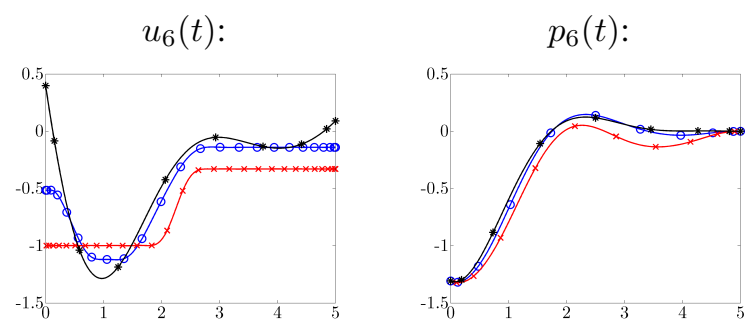

Fig. 4. Optimal control and position trajectories for the mass indexed by $n=6$ in a mass-spring system with $N=10$ masses and $\gamma=0.1$ $(\circ), \gamma=1(\times)$, and standard LQR $(*)$.
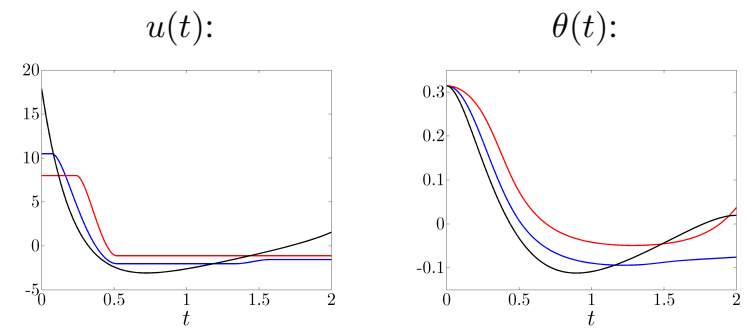

Fig. 5. Optimal control and angle trajectories for the inverted pendulum with $\gamma=1$ (blue), $\gamma=10$ (red), and standard LQR (black).

optimal control signal and the trajectory of the angle of the pendulum $\theta$ are shown in Fig. 5. As $\gamma$ increases, the total variation of the control signal decreases. This results in a larger deviation of the angle of the pendulum.

\section{CONCLUDING REMARKS}

In this paper, we consider the optimal control design that aims at balancing the quadratic performance with sparsity and total variation of the control signal. We show that this convex optimization problem amounts to solving a two point boundary value problem with non-differentiable nonlinearities. Furthermore, we use the ADMM algorithm to compute the optimal controller by solving a sequence of linear TPBVPs. Parallel developments for discrete-time systems that were part of our original submission will be reported elsewhere.

While we have demonstrated flexibility of ADMM, efficiency of the proposed algorithm is to be tested against state-of-the-art two point boundary value solvers. In addition, it is also of interest to investigate the effectiveness of the developed approach in applications that give rise to large problems.

\section{REFERENCES}

[1] S. Boyd, M. Mueller, B. O'Donoghue, and Y. Wang, "Performance bounds and suboptimal policies for multi-period investment," Foundations and Trends in Optimization, 2013, to appear.

[2] Y. Ma, A. Kelman, A. Daly, and F. Borrelli, "Predictive control for energy efficient buildings with thermal storage: Modeling, simulation, and experiments," IEEE Control Systems Magazine, vol. 32, no. 1, pp. 44-64, 2012.

[3] C. V. Rao, S. J. Wright, and J. B. Rawlings, "Application of interior-point methods to model predictive control," Journal of Optimization Theory and Applications, vol. 99, no. 3, pp. 723757, 1998
[4] Y. Wang and S. Boyd, "Fast model predictive control using online optimization," IEEE Transactions on Control Systems Technology, vol. 18, no. 2, pp. 267-278, 2010.

[5] S. Richter, C. N. Jones, and M. Morari, "Towards computational complexity certification for constrained MPC based on Lagrange relaxation and the fast gradient method," in Proceedings of the 50th IEEE Conference on Decision and Control and European Control Conference, 2011, pp. 5223-5229.

[6] J. Mattingley, Y. Wang, and S. Boyd, "Receding horizon control: Automatic generation of high-speed solvers," IEEE Control Systems Magazine, vol. 31, no. 3, pp. 52-65, 2011.

[7] S. Richter, C. N. Jones, and M. Morari, "Computational complexity certification for real-time MPC with input constraints based on the fast gradient method," IEEE Tran. Automat. Control, vol. 57, no. 6, pp. 1391-1403, 2012.

[8] A. Domahidi, A. U. Zgraggen, M. N. Zeilinger, M. Morari, and C. N. Jones, "Efficient interior point methods for multistage problems arising in receding horizon control," in Proceedings of the 51st IEEE Conference on Decision and Control, 2012, pp. 668-674.

[9] B. O'Donoghue, G. Stathopoulos, and S. Boyd, "A splitting method for optimal control," IEEE Transactions on Control Systems Technology, 2013, in press.

[10] B. O'Donoghue, "Suboptimal control policies via convex optimization," Ph.D. dissertation, Stanford, 2012.

[11] F. Farokhi, I. Shames, and K. H. Johansson, "Distributed MPC via dual decomposition and alternative direction method of multipliers," arXiv:1207.3178, 2012.

[12] S. Boyd, N. Parikh, E. Chu, B. Peleato, and J. Eckstein, "Distributed optimization and statistical learning via the alternating direction method of multipliers," Foundations and Trends in Machine Learning, vol. 3, no. 1, pp. 1-124, 2011.

[13] F. Lin, M. Fardad, and M. R. Jovanović, "Sparse feedback synthesis via the alternating direction method of multipliers," in Proceedings of the 2012 American Control Conference, Montréal, Canada, 2012, pp. 4765-4770.

[14] F. Lin, M. Fardad, and M. R. Jovanović, "Design of optimal sparse feedback gains via the alternating direction method of multipliers," IEEE Trans. Automat. Control, 2013, doi:10.1109/TAC.2013.2257618; also arXiv:1111.6188.

[15] F. Lin, M. Fardad, and M. R. Jovanović, "Algorithms for leader selection in large dynamical networks: noise-corrupted leaders," in Proceedings of the 50th IEEE Conference on Decision and Control and European Control Conference, Orlando, FL, 2011, pp. 2932-2937.

[16] F. Lin, M. Fardad, and M. R. Jovanović, "Algorithms for leader selection in stochastically forced consensus networks," IEEE Trans. Automat. Control, 2013, conditionally accepted; also arXiv: 1302.0450

[17] N. Dhingra, F. Lin, M. Fardad, and M. R. Jovanović, "On identifying sparse representations of consensus networks," in Preprints of the 3rd IFAC Workshop on Distributed Estimation and Control in Networked Systems, Santa Barbara, CA, 2012, pp. $305-310$

[18] M. Fardad, X. Zhang, F. Lin, and M. R. Jovanović, "On the optimal dissemination of information in social networks," in Proceedings of the 51th IEEE Conference on Decision and Control, Maui, HI, 2012, to appear.

[19] F. Dörfler, M. R. Jovanović, M. Chertkov, and F. Bullo, "Sparse and optimal wide-area damping control in power networks," in Proceedings of the 2013 American Control Conference, Washington, DC, 2013, to appear.

[20] M. Annergren, A. Hansson, and B. Wahlberg, "An ADMM algorithm for solving $\ell_{1}$ regularized MPC," in Proceedings of 51 st Conference on Decision and Control, Maui, HI, 2012, pp. 4486-4491.

[21] S. Boyd and L. Vandenberghe, Convex Optimization. Cambridge University Press, 2004.

[22] E. J. Candès, M. B. Wakin, and S. P. Boyd, "Enhancing sparsity by reweighted $\ell_{1}$ minimization," Journal of Fourier Analysis and Applications, vol. 14, pp. 877-905, 2008.

[23] H. Kwakernaak and R. Sivan, Linear optimal control systems. Wiley-Interscience, 1972.

[24] L. N. Trefethen, N. Hale, R. B. Platte, T. A. Driscoll, and R. Pachón, "Chebfun version 4," University of Oxford, 2011, http://www.maths.ox.ac.uk/chebfun/. 